than contralateral motor cortex, when it is excited subliminally beforehand.

\title{
C3. Clinical Seizure Pattern and Spreading of the Induced Epileptic Discharges from the Frontal Lobe in Man.
}

\author{
T. WADA
}

Dept. of Neuropsy., Hirosaki Univ. School of Med.

As clinical seizure symptom occurred in the 20 cases with definite epileptic EEG-focus localized in the frontal lobe, including 3 cases with rhythmic spikeand-wave complex, which were chosen out of ca. 500 epileptics treated during the past years, the followings were predominant: an adversive movement, especially a contraverse, and a disturbance of consciousness with tonic and/or arrest state. Whether those are characteristic to the frontal lobe or not, the author has anew reconsidered the following results had been obtained by himself in the past.

In 20 schizophrenia the so-called electrostimography had been carried out under unanaesthetised condition by means of the implanted multi-needle electrode method to various cortical and subcortical portions of the frontal lobe and thalamic region. If the stimulating electrical current were gradually increased, all cases showed contraversive tonic posture with arrest state, and finally fell down into focal beginning convulsion with unconsciousness. If the focal discharge localized in a certain portion were remained, there occurred neither unconsciousness nor convulsion. However, when the induced discharge spreaded into the total frontal, especially into the thalamus, accompanying the after-discharge with diffuse dysrhythmia, it seemed to occur a definite loss of consciousness. As far as such consciousness condition were concerned, it differs clearly from the automatism due to the temporal lobe epilepsy: in case of the frontal lobe, it is not a free complex behavior but a arrest-tonic state. Accordingly, in the frontal lobe epilepsy, it should be important to notice the arrest-tonic state as well as the contraversive movement as special symptoms.

Recently the author has begun such a treatment with Resochin in combination with other antiepileptic drugs, in which it seems to be effective for control of arrest seizure or abortive tonic fit than convulsive one, accompanying a remarkable normalization of abnormal EEG discharges. In refer to such findings, the author is likely to assume that Resochin might prevent an out-burst of epileptic discharges spreading into the subcortical tissue and the thalamic region (vig. the deep centrencephalic level). 